\begin{tabular}{l} 
O P E R A T I O N S R E S E A R C H A N D D E C I S I O N S \\
\hline No. 4
\end{tabular}

DOI: $10.37190 /$ ord200401

\title{
IMPATIENT CUSTOMERS IN MARKOVIAN QUEUE WITH BERNOULLI FEEDBACK AND WAITING SERVER UNDER VARIANT WORKING VACATION POLICY
}

\author{
AMINA ANGELIKA BOUCHENTOUF ${ }^{1 *}$, LAHCENE YAHIAOUI $^{2}$, \\ MOKHTAR KADI ${ }^{2}$, SHAKIR MAJID ${ }^{3}$ \\ ${ }^{1}$ Department of Mathematics, Djillali Liabes University of Sidi Bel Abbes, \\ BP 89 Sidi Bel Abbes 22000-Algeria \\ ${ }^{2}$ The University Moulay Tahar of Saida, BP 138 cité ENNASR 20000, Saida, Algeria \\ ${ }^{3}$ Government Degree College Mendhar, Poonch, Jammu and Kashmir, India
}

\begin{abstract}
This paper deals with customers' impatience behaviour for single server Markovian queueing system under $K$-variant working vacation policy, waiting server, Bernoulli feedback, balking, reneging, and retention of reneged customers. Using the probability generating function (PGF) technique, we obtain the steady-state solution of the system. Besides, we prove the stochastic decomposition properties. Useful performance measures of the considered queueing system are derived. A cost model is developed. Then, the parameter optimisation is carried out numerically, using a quadratic fit search method (QFSM). Finally, numerical examples are provided to visualise the analytical results.
\end{abstract}

Keywords: queueing models, variant of a multiple working vacation, balking, reneging, retention of reneged customers, simulation

\section{Introduction}

In queueing theory, working vacation queues have long been subject to intensive research due to their important application in many areas, particularly in the areas of mailing service, file transfer, and network service. The pioneering research work on the field was done by Servi and Finn [21]. In the literature, extensive analyses have been carried out of two types of working vacation policies, including single and multiple working vacations. In single working vacation, after coming back from vacation, if there

*Corresponding author, email address: bouchentouf.amina1@gmail.com Received 8 April 2020, accepted 13 December 2020 
are some customers in the system, the server immediately starts the service, otherwise, he remains idle in the system, waiting for a new arrival [27, 26, 22]. In multiple working vacations, the server resumes several working vacation each time the system leads to an empty state [14, 20,23]. Recently, a new concept of working vacation policy has been introduced, where the server can take some fixed number of consecutive vacations if the system remains empty at the end of a working vacation $[36,35,31]$. Further, in various life situations, once the busy period is ended, the server has to wait an amount of time before it goes on vacation. Many authors have dealt with this sort of vacation queues (e.g., [30, 15, 17, 1, 10, 2]).

One further aspect broadly considered in queueing systems is Bernoulli's feedback of customers. Diverse queueing situations have the distinction that customers can be served repeatedly for some reasons. When a customer's service is not satisfied, it can be retried many times until the service is successful. ARQ (Automatic Repeat Re-Quest) protocol, LAN (Local Area Network), and SMTP (Simple Mail Transfer Protocol) are perfect applications of this model. A few interesting papers include Takacs [28], Krishnakumar et al. [19], Choudhury and Paul [9], Kalidass and Kasturi [16], Varalakshmi et al. [34], Varalakshmi et al. [33], Bouchentouf and Messabihi [7], Bouchentouf et al. [6] and the references therein.

Customer's impatience is a prominent feature in queueing theory. Over the last decades, increasing attention can be seen on working vacation queues with impatient customers. These are widely studied due to their large applicability for modelling purpose of call centres, computer and telecommunication systems, as well as production and manufacturing system. Yue et al. [32] deal with a single server Markovian queue with working vacation and impatient customers. Laxmi and Jyothsna [11] treat a finite buffer renewal input queueing model with balking and multiple working vacations. Laxmi and Rajesh [12] analyse a variant working vacations queue with customers' impatience. Then, batch arrival queueing model with variant working vacations with reneging is investigated in Laxmi and Rajesh [13]. Bouchentouf and Yahiaoui [8] obtain steady-state probabilities of a queueing model with multiple working vacations, vacation interruption, reneging, and Bernoulli feedback. Further, Majid and Manoharan [24] study the impatience behaviour of customers in a multi-server Markovian queue with single and multiple working vacation policies. For some recent papers on working vacation queues with customers impatience, we cite Sudhesh and Azhagappan [25], Yahiaoui et al. [29], Bouchentouf and Guendouzi [4], Bouchentouf et al. [5], Azhagappan [2], and Bouchentouf and Gunedouzi [3] and the references therein.

The remainder of the paper is organised as follows. In Section 2, we describe the model and present the balance equations of the considered queueing model. In Section 3, the steady-state probabilities of the system are explicitly derived. In Section 4, we present the stochastic decomposition theorems. In Section 5, useful performance measures are derived. Then, Section 6 is dedicated to a few special cases. A cost model is developed in Section 7. In Section 8, the parameter optimisation is carried out numerically, using a quadratic fit 
search method (QFSM). Further, numerical examples are given to visualise the impact of different system parameters on the characteristics of the queueing model. In Section 9, the research work is concluded.

\section{The model}

We develop a model for an $M / M / 1$ queue with $K$-variant working vacation, waiting server, Bernoulli feedback, and impatient customers. The detailed description of the model is as follows:

- The customers arrive at the system, according to a Poisson process of rate $\lambda$. There is an infinite waiting space. The service discipline is First Come First Served (FCFS).

- The service time during a regular busy period is denoted by the random variable exponentially distributed with a parameter $\mu$.

- Once the regular busy period is finished, the server waits a random period before taking a working vacation, this waiting time is supposed to be exponentially distributed with a parameter $\eta$. When the duration of the waiting server expires, the server leaves for working vacation which follows an exponential distribution with parameter $\phi$. During this period, the server provides service at a lower rate if no customer arrives. The service times are exponentially distributed with a parameter $v$, where $v<\mu$. At the working vacation completion instant, if some customers are found in the system, the server switches to the regular busy period, otherwise, it takes a finite number, namely $K$, of successive working vacations. When the $K$ consecutive working vacations are complete, the server comes back to the regular busy period and, depending on the arrival of new customers, it stays idle or busy.

- During the working vacation period, each new arrival starts up an impatience timer independently of the other customers in the queue, which is assumed to be exponentially distributed with parameter $\xi$. The impatient customers may leave the system with probability $\alpha$. The latter can be retained in the system with probability $\alpha^{\prime}=1-\alpha$.

- A customer who on arrival finds at least one customer in the system either decides to enter the queue with probability $\theta$ or to balk with probability $\bar{\theta}=1-\theta$.

- If, after completion of service, a customer is not satisfied with its quality, they can come back to the system with some probability $\beta^{\prime}$ for another service, or decide to leave the system with probability where $\beta+\beta^{\prime}=1$.

- The inter-arrival times, waiting server times, working vacation times, service times, and impatience times are independent of each other.

This model is suggested with practical motivation. We consider a healthcare system with a chief physician (the principal server) and a physician assistant. The patients enter the system for some medical consultations. During the regular busy period, it is the head physician who treats the patients. At the end of this period, the chief physician waits 
a while before going on vacation. During the absence, she/he can be replaced by a physician assistant (working vacation). Naturally, during this time, the patients are served at a lower rate. Once the working vacation period is ended, the chief physician medical returns to regular working period if some patients are waiting in the queue; otherwise (s)he can take fixed consecutive vacations. At the end of the successive vacations, the chief physician comes back to the regular working period and remains inactive or busy, depending on the availability of patients in the system. During the working vacation period, a patient in the system may get impatient and abandon before being served if the queue becomes too long. The patients can be convicted to stay in the system. This could be done either by increasing the service rate of a physician assistant or by asking other physician assistants to provide services during the absence of the chief physician. A patient who cannot be directly helped by a chief physician and/or assistant physician may be informed of the wait time she/he has to experience before she/he will be serviced. Then the patient can hang up (i.e., balk) or decide to enter the system. Moreover, the patients may be unhappy with the service for some reasons. In this case, they can rejoin the system as feedback customers to get another consultation or complete their one.

Suppose that $\{L(t), t \geq 0\}$ denotes the number of customers in the system at a time $t$ and let $S(t)$ be the status of the server at a time $t$, where

$$
S(t=) \begin{cases}j \quad \text { when the server is taking the }(j+1) \text { th working vacation at time } t \\ j=\overline{0, K-1} \\ K \quad \text { the server is in busy period at time } t\end{cases}
$$

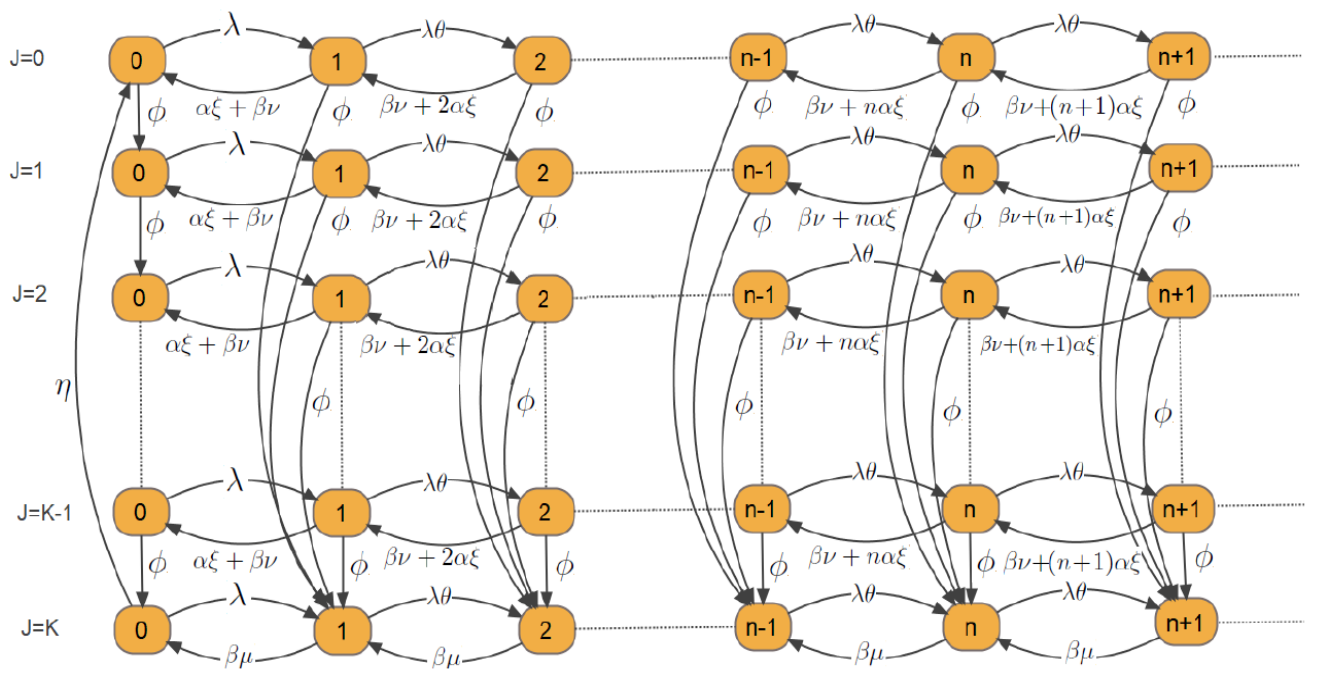

Fig. 1. State transition rate diagram of the queueing model 
Then, $\{(S(t), L(t)), t \geq 0\}$ is a continuous-time Markov chain on the state space $\Omega=\{(j, n), n \geq 0$ and $j=\overline{0, K}\}$.

Let $P_{j, n}=\lim _{t \rightarrow \infty} P\{S(t)=j, L(t)=n\}$ denote the system state probabilities of the process $\{(S(t), L(t)), t \geq 0\}$. The state transition diagram corresponding to our queueing system is depicted in Fig. 1.

Then, $P_{j, n}$ for $n \geq 0, j=\overline{0, K}$ satisfy the Chapman-Kolmogorov equations as follows:

$$
\begin{gathered}
(\lambda+\phi) P_{0,0}=(\alpha \xi+\beta v) P_{0,1}+\eta P_{K, 0} \\
(\lambda+\phi) P_{j, 0}=(\alpha \xi+\beta v) P_{j, 1}+\phi P_{j-1,0}, j=\overline{1, K-1} \\
(\lambda \theta+\phi+\beta v+\alpha \xi) P_{j, 1}=\lambda P_{j, 0}+(\beta v+2 \alpha \xi) P_{j, 2}, j=\overline{0, K-1} \\
(\lambda \theta+\phi+\beta v+n \alpha \xi) P_{j, n}=\lambda \theta P_{j, n-1}+(\beta v+(n+1) \alpha \xi) P_{j, n+1}, n \geq 2, j=\overline{0, K-1} \\
(\lambda+\eta) P_{K, 0}=\beta \mu P_{K, 1}+\phi P_{K-1,0} \\
(\lambda \theta+\beta \mu) P_{K, 1}=\lambda P_{K, 0}+\beta \mu P_{K, 2}+\phi \sum_{j=0}^{K-1} P_{j, 1} \\
(\lambda \theta+\beta \mu) P_{K, n}=\lambda \theta P_{K, n-1}+\beta \mu P_{K, n+1}+\phi \sum_{j=0}^{K-1} P_{j, n}, n \geq 2
\end{gathered}
$$

The normalising condition is given as

$$
\sum_{n=0}^{\infty} \sum_{j=0}^{K} P_{j, n}=1
$$

\section{Steady-state solution}

Consider the partial probability generating functions (PGFs) as follows:

$$
G_{j}(z)=\sum_{n=0}^{\infty} P_{j, n} z^{n}, \quad 0 \leq z \leq 1
$$


and let

$$
G_{j}^{\prime}(z)=\frac{d}{d z} G_{j}(z)=\sum_{n=1}^{\infty} n P_{j, n} z^{n-1}, \quad j=\overline{0, K}
$$

Multiplying equation (4) by $z^{n}$, summing all possible values of $n$, and using equations (1)-(3), we find

$$
\begin{aligned}
\alpha \xi(1-z) z G_{j}^{\prime}(z) & -[(\lambda \theta z-\beta v)(1-z)+\phi z] G_{j}(z) \\
& =-z \Delta_{j}+(\beta v+\lambda \bar{\theta} z)(1-z) P_{j, 0}, 0 \leq j \leq K-1
\end{aligned}
$$

This equation can be rewritten as

$$
\begin{aligned}
G_{j}^{\prime}(z) & -\left(\frac{\lambda \theta}{\alpha \xi}-\frac{\beta v}{\alpha \xi z}+\frac{\phi}{\alpha \xi(1-z)}\right) G_{j}(z) \\
& =-\frac{\Delta_{j}}{\alpha \xi(1-z)}+\left(\frac{\beta v}{\alpha \xi_{z}}+\frac{\lambda \bar{\theta}}{\alpha \xi}\right) P_{j, 0}, 0 \leq j \leq K-1
\end{aligned}
$$

where

$$
\Delta_{j}= \begin{cases}\eta P_{K, 0}, & j=0 \\ \phi P_{j-1,0}, & 1 \leq j \leq K-1\end{cases}
$$

Similarly, from equations (5)-(7), we get

$$
\begin{aligned}
((\lambda \theta z & -\beta \mu)(1-z)) G_{K}(z) \\
& =\phi z \sum_{j=0}^{K-1} G_{j}(z)-\phi z \sum_{j=0}^{K-2} P_{j, 0}-((\lambda \bar{\theta} z+\beta \mu)(1-z)+\eta z) P_{K, 0}
\end{aligned}
$$

Equivalently, for $z \neq 1$, we have

$$
G_{K}(z)=\phi z \frac{\sum_{j=0}^{K-1} G_{j}(z)-\left(\sum_{j=0}^{K-2} P_{j, 0}+\frac{\eta}{\phi} P_{K, 0}\right)}{(\lambda \theta z-\beta \mu)(1-z)}-\frac{\lambda \bar{\theta} z+\beta \mu}{\lambda \theta z-\beta \mu} P_{K, 0}
$$


By solving the differential equation (12), we obtain

$$
\begin{aligned}
G_{j}(z)= & \exp \left(\frac{\lambda \theta z}{\alpha \xi}\right) z^{-\frac{\beta v}{\alpha \xi}}(1-z)^{-\phi / \alpha \xi}\left(\frac{-\Delta_{j}}{\alpha \xi} K_{0}(z)+\left(\frac{\beta v}{\alpha \xi} K_{1}(z)\right.\right. \\
& \left.\left.+\frac{\lambda \bar{\theta}}{\alpha \xi} K_{2}(z)\right) P_{j, 0}\right), 0 \leq j \leq K-1
\end{aligned}
$$

where

$$
\begin{aligned}
& K_{0}(z)=\int_{0}^{z} \exp \left(-\frac{\lambda \theta t}{\alpha \xi}\right) t^{\beta v / \alpha \xi}(1-t)^{(\phi / \alpha \xi)-1} d t \\
& K_{1}(z)=\int_{0}^{z} \exp \left(-\frac{\lambda \theta t}{\alpha \xi}\right) t^{(\beta v / \alpha \xi)-1}(1-t)^{\phi / \alpha \xi} d t
\end{aligned}
$$

and

$$
K_{2}(z)=\int_{0}^{z} \exp \left(-\frac{\lambda \theta t}{\alpha \xi}\right) t^{\beta \nu / \alpha \xi}(1-t)^{\phi / \alpha \xi} d t
$$

Next, to get $P_{K, 0}, P_{j, 0}$ and $\sum_{j=0}^{K-1} G_{j}(1)$ in terms of $P_{0,0}$, we take $z=1$ in equations (15) and equation (13), respectively (according to the property of PGF, i.e., the continuity), we obtain

$$
P_{K, 0}=\frac{A}{\eta} P_{0,0}, j=0
$$

and

$$
P_{j, 0}=C P_{j-1,0}=C^{j} P_{0,0}, j=1, \ldots, K-1
$$

where

$$
A=\frac{\beta v K_{1}(1)+\lambda \bar{\theta} K_{2}(1)}{K_{0}(1)}, C=\frac{\phi}{A}
$$


and

$$
\sum_{j=0}^{K-1} G_{j}(1)=\sum_{j=0}^{K-2} P_{j, 0}+\frac{\Delta_{0}}{\phi}
$$

Now, substituting equations (16) and (17) into equation (18), we have

$$
\sum_{j=0}^{K-1} G_{j}(1)=H P_{0,0}
$$

where

$$
H=\frac{1-C^{K}}{(1-C) C}
$$

To get $G_{0}(1), G_{j}(1)$ and $G_{j}^{\prime}(1)$ in term of $P_{0,0}$, we put $z=1$ in equations (11) and (12), respectively. Thus,

$$
G_{j}(1)=C^{j-1} P_{0,0}, 0 \leq j \leq K-1
$$

and

$$
G_{j}^{\prime}(1)=\frac{(\lambda \theta-\beta v) C^{-1}+\beta v+\lambda \bar{\theta}}{\alpha \xi+\phi} C^{j} P_{0,0}, 0 \leq j \leq K-1
$$

Finally, to get $G_{K}(1)$ in term of $P_{0,0}$, we put $z=1$ in equation (14)

$$
G_{K}(1)=\frac{\phi}{\beta \mu-\lambda \theta} \sum_{j=0}^{K-1} G_{j}^{\prime}(1)-\frac{\lambda \bar{\theta}+\beta \mu}{\lambda \theta-\beta \mu} P_{K, 0}
$$

To get $G_{K}(1)$ into term of $P_{0,0}$, substituting equations (16), (22) into equation (23),

$$
G_{K}(1)=H_{K} P_{0,0}
$$

where 


$$
H_{K}=\frac{\phi\left((\lambda \theta-\beta v) C^{-1}+\beta v+\lambda \bar{\theta}\right)\left(1-C^{K}\right)}{(\beta \mu-\lambda \theta)(\alpha \xi+\phi)(1-C)}-\frac{(\lambda \bar{\theta}+\beta \mu) A}{(\lambda \theta-\beta \mu) \eta}
$$

Now, using normalisation condition, we easily obtain

$$
P_{0,0}=\left(H+H_{K}\right)^{-1}
$$

where $H$ and $H_{K}$ are given by equations (20) and (25), respectively. This completes the evaluation of steady-state probabilities.

\section{Stochastic decomposition}

If $\rho=\frac{\lambda \theta}{\beta \mu}<1$, then the steady-state queue length can be decomposed into the sum of independent random variables: $L=L_{b}+L_{w v}$, where $L_{b}$ is the steady-state queue length of the system without working vacation and $L_{w v}$ is the additional queue length due to the effect of working vacation with its probability generating function

$$
L_{w v}(z)=\frac{1}{1-\rho}\left(\left(1-\rho z-\frac{\phi z}{\beta \mu(1-z)}\right) \sum_{j=0}^{K-1} G_{j}(z)+\phi z \frac{\sum_{j=0}^{K-1} G_{j}(1)}{\beta \mu(1-z)}+\frac{\lambda \bar{\theta} z+\beta \mu}{\beta \mu} P_{K, 0}\right)
$$

Proof. Consider

$$
\begin{aligned}
G(z)= & \sum_{j=0}^{K-1} G_{j}(z)+G_{K}(z)=\left(1+\frac{\phi z}{(\lambda \theta z-\beta \mu)(1-z)}\right) \sum_{j=0}^{K-1} G_{j}(z) \\
& -\phi z \frac{\sum_{j=0}^{K-1} G_{j}(1)}{(\lambda \theta z-\beta \mu)(1-z)}-\frac{\lambda \bar{\theta} z+\beta \mu}{\lambda \theta z-\beta \mu} P_{K, 0} \\
= & \left(\frac{\beta \mu-\lambda \theta}{\beta \mu-\lambda \theta z}\right)\left(\left(\frac{\beta \mu-\lambda \theta z}{\beta \mu-\lambda \theta}-\frac{\phi z}{(\beta \mu-\lambda \theta)(1-z)}\right) \sum_{j=0}^{K-1} G_{j}(z)\right. \\
& \left.+\phi z \frac{\sum_{j=0}^{K-1} G_{j}(1)}{(\beta \mu-\lambda \theta)(1-z)}+\frac{\lambda \bar{\theta} z+\beta \mu}{\lambda \theta z-\beta \mu} P_{K, 0}\right)=\left(\frac{1-\rho}{1-\rho z}\right) L_{w v}(z)
\end{aligned}
$$


where $L_{w v}(z)$ is the PGF of the additional queue length due to the effect of working vacation and is expressed as

$$
\begin{aligned}
L_{w v}(z)= & \frac{1}{1-\rho}\left(\sum_{j=0}^{K-1}\left(\sum_{n=0}^{\infty} P_{j, n} z^{n}-\rho \sum_{n=1}^{\infty} P_{j, n-1} z^{n}+\frac{\phi}{\beta \mu} \sum_{n=1}^{\infty} \sum_{i=0}^{\infty} P_{j, n+i} z^{n}\right)\right. \\
& \left.+\left(\frac{\lambda \bar{\theta} z}{\beta \mu}+1\right) \frac{A}{\eta} P_{0,0}\right)=\sum_{n=0}^{\infty} t_{n} z^{n}
\end{aligned}
$$

where

$$
\begin{aligned}
& t_{0}=\frac{1}{1-\rho}\left(\sum_{j=0}^{K-1} P_{j, 0}+\frac{A}{\eta} P_{0,0}\right) \\
& t_{1}=\frac{1}{1-\rho}\left(\sum_{j=0}^{K-1}\left(P_{j, 1}-\rho P_{j, 0}+\frac{\phi}{\beta \mu} \sum_{i=0}^{\infty} P_{j, 1+i}\right)+\frac{\lambda \bar{\theta} A}{\beta \mu \eta} P_{0,0}\right) \\
& t_{n}=\frac{1}{1-\rho}\left(\sum_{j=0}^{K-1}\left(P_{j, n}-\rho P_{j, n-1} \frac{\phi}{\beta \mu} \sum_{i=0}^{\infty} P_{j, n+i}\right)\right), n \geq 2
\end{aligned}
$$

Next, we show that $\sum_{n=0}^{\infty} t_{n}=1$ for $t_{n} \in[0,1]$

$$
\begin{aligned}
\sum_{n=0}^{\infty} t_{n} & =\frac{1}{1-\rho}\left(\sum_{j=0}^{K-1}\left(\sum_{n=0}^{\infty} P_{j, n}-\rho \sum_{n=1}^{\infty} P_{j, n-1}+\frac{\phi}{\beta \mu} \sum_{n=1}^{\infty} \sum_{i=0}^{\infty} P_{j, n+i}\right)+\left(\frac{\lambda \bar{\theta}}{\beta \mu}+1\right) \frac{A}{\eta} P_{0,0}\right) \\
& =\frac{1}{1-\rho}\left((1-\rho) \sum_{j=0}^{K-1}\left(\sum_{n=0}^{\infty} P_{j, n}+\frac{\phi}{\beta \mu} \sum_{n=1}^{\infty} \sum_{i=0}^{\infty} n P_{j, n}\right)+\left(\frac{\lambda \bar{\theta}}{\beta \mu}+1\right) \frac{A}{\eta} P_{0,0}\right)
\end{aligned}
$$

Using equation (23) and the normalisation condition in equation (27), we obtain

$$
\begin{aligned}
\sum_{n=0}^{\infty} t_{n}= & \frac{1}{1-\rho}\left((1-\rho) \sum_{j=0}^{K-1} \sum_{n=0}^{\infty} P_{j, n}+\left(\frac{\lambda \bar{\theta}}{\beta \mu}+1\right) \frac{A}{\eta} P_{0,0}\right. \\
& \left.+\frac{1}{\beta \mu}\left((\beta \mu-\lambda \theta)\left(1-\sum_{j=0}^{K-1} G_{j}(1)\right)-(\lambda \bar{\theta}+\beta \mu) \frac{A}{\eta} P_{0,0}\right)\right) \\
& =\sum_{j=0}^{K-1} G_{j}(1)+\frac{1}{1-\rho}(1-\rho)\left(1-\sum_{j=0}^{K-1} G_{j}(1)\right)=1
\end{aligned}
$$


Hence, $L_{w v}(z)$ is the PGF of the additional queue length due to the working vacation. If $\rho<1$, then the steady-state waiting time $W$ can be decomposed into the sum of independent random variables: $W=W_{b}+W_{w v}$, where $W_{b}$ is the stationary waiting time of customers in the system without working vacation, and $W_{w v}$ is the additional stationary waiting time due to the effect of working vacation and has a distribution with its Laplace Stieltjes transform (LST):

$$
\begin{aligned}
W_{w s}^{*}(z)= & \frac{1}{(\beta \mu-\lambda \theta) s}\left(\{(\beta \mu-\lambda \theta+\theta s) s-\phi(\lambda-s)\} \sum_{j=0}^{k-1} G_{j}\left(1-\frac{s}{\lambda}\right)\right. \\
& \left.+\phi(\lambda-s) \sum_{j=0}^{k-1} G_{j}(1)+s\left(\lambda \bar{\theta}\left(1-\frac{s}{\lambda}\right)+\beta \mu\right)\right)
\end{aligned}
$$

Proof. It is well known that the classical relationship between the PGF of $L$ and the LST of waiting time [18] is given as

$$
L(z)=W^{*}(\lambda(1-z))
$$

Assume that $s=\lambda(1-z)$. Therefore, substitute $z=1-\frac{s}{\lambda}$ and $1-z=\frac{s}{\lambda}$. Applying these relations in (27), we easily derive the desired result.

Remark. We suppose that the system is modelled by a queue with infinite capacity, and that arrival rate $\lambda$, and service rate $\mu$ exist. The customers enter the system with probability $\theta$ and leave the system after getting service with probability $\beta$. We suppose that customers' impatience occurs because of the unavailability of the server, that is the queue during a regular busy period is considered as a classical $M / M / 1$ queue with balking and feedback. Evidently, during this period, for the steady-state conditions to exist, we must have $\theta \lambda<\beta \mu$. This is the condition for the stability of our system. When the mean number of arrivals in the system is greater than the maximum number of customer that the system can serve, i.e., $\beta \mu<\theta \lambda$, it means that the size of the queue never stabilises and there is no stable state. However, during the working vacation period, we do not have such a problem, and even when $\beta \mu<\theta \lambda$, the stationary queue length distribution exists. Consequently, we can say that the considered queueing system is stable under the condition $\theta \lambda / \beta \mu<1$.

When $\theta \lambda=\beta \mu$, the randomness will prevent the queue from being emptied, which generates unlimited growth of the queue. In such a case, the steady state exists only if the arrivals and the service are deterministic and well scheduled. 


\section{Performance measures}

In this section, we derive some important performance measures.

- Mean number of customers in the system $\left(E\left(L_{s}\right)\right)$.

Let $E\left[L_{j}\right], 0 \leq j \leq K-1$ be the mean system size when the server is taking a vacation $j+1$, and let $E\left[L_{K}\right]$ be the mean system size when the server is busy. Thus, the mean number of customers in the system is as follows:

$$
E\left(L_{s}\right)=E\left[L_{K}\right]+E\left[L_{w v}\right]
$$

From equation (22), we have

$$
E\left[L_{j}\right]=G_{j}^{\prime}(1)=\frac{(\lambda \theta-\beta v) C^{-1}+\beta v+\lambda \bar{\theta}}{\alpha \xi+\phi} C^{j} P_{0,0}, 0 \leq j \leq K-1
$$

Therefore, the mean system size when the server is on WV, denoted by $E\left[L_{w v}\right]$ is obtained as

$$
E\left[L_{w v}\right]=\sum_{j=0}^{K-1} G_{j}^{\prime}(1)=\left(\frac{\lambda \theta-\beta v}{\alpha \xi+\phi}+C \frac{\lambda \bar{\theta}+\beta v}{\alpha \xi+\phi}\right) \frac{1-C^{K}}{C(1-C)} P_{0,0}
$$

where $P_{0,0}$ is given by equation (26). Next, by deriving equation (13) and taking $z=1$, we find

$$
\begin{aligned}
E\left[L_{K}\right]=G_{K}^{\prime}(1)= & \frac{\phi}{\beta \mu-\lambda \theta}\left(\left(\frac{\beta \mu}{\beta \mu-\lambda \theta}+\frac{\lambda \theta-(\phi+\beta \nu+\alpha \xi)}{2 \alpha \xi+\phi}\right) \times \sum_{j=0}^{K-1} G_{j}^{\prime}(1)\right. \\
& \left.+\left(\frac{\lambda(\theta+C \bar{\theta})\left(1-C^{K}\right)}{(2 \alpha \xi+\phi) C(1-C)}+\frac{\lambda \bar{\theta} \beta \mu A}{(\beta \mu-\lambda \theta)^{2} \eta}\right) P_{0,0}\right)
\end{aligned}
$$

where $G_{j}(1), 0 \leq j \leq K-1$ is given by equation (21) and $P_{0,0}$ by equation (26).

A mean number of customers in a queue $\left(E\left(L_{\mathrm{q}}\right)\right)$.

$$
E\left[L_{q}\right]=\sum_{j=0}^{K} \sum_{n=1}^{\infty}(n-1) P_{j, n}=E\left[L_{w v}\right]+E\left[L_{K}\right]-\sum_{j=0}^{K}\left(G_{j}(1)-P_{j, 0}\right)
$$


The probability that the server is on vacation $\left(P_{w v}\right)$

$$
P_{w v}=\sum_{j=0}^{K-1} G_{j}(1)=\frac{1-C^{K}}{(1-C) \times C} P_{0,0}
$$

where $P_{0,0}$ is given by equation (26).

The probability that the server is busy $\left(P_{b}\right)$

$$
P_{b}=1-P_{K, 0}-P_{w v}=1-\left(\frac{A}{\eta}+\frac{1-C^{K}}{(1-C) \times C}\right) P_{0,0}
$$

The probability that the server is idle during a busy period $\left(P_{i d}\right)$

$$
P_{i d}=P_{K, 0}
$$

Mean number of customers served $\left(S_{r}\right)$

$$
S_{r}=\beta \mu P_{b}+\beta v \sum_{j=0}^{K-1}\left(G_{j}(1)-P_{j, 0}\right)
$$

Average reneging rate $\left(R_{\text {ren }}\right)$

$$
R_{r e n}=\alpha \xi \sum_{j=0}^{K-1} \sum_{n=1}^{\infty} n P_{j, n}=\alpha \xi E\left(L_{w v}\right)
$$

where $E\left(L_{w v}\right)$ is given by equation (30). Average retention rate $\left(R_{r e t}\right)$

$$
R_{r e t}=\bar{\alpha} \xi \sum_{j=0}^{K-1} \sum_{n=1}^{\infty} n P_{j, n}=\bar{\alpha} \xi E\left(L_{w v}\right)
$$

The average balking rate $\left(R_{b}\right)$

$$
R_{b}=\sum_{j=0}^{K} \sum_{n=1}^{\infty} \lambda \bar{\theta} P_{j, n}=\lambda \bar{\theta}\left(1-\sum_{j=0}^{K} P_{j, 0}\right)=\lambda \bar{\theta}\left(1-\left(\frac{1-C^{K}}{1-C}+\frac{A}{\eta}\right) P_{0,0}\right)
$$

The average rate of loss customers $\left(L_{r}\right)$

$$
L_{r}=R_{r e n}+B_{r}
$$




\section{Special cases}

When $v=0, K=1, \theta=1, \alpha=1$, and $\beta=1$, then the steady-state probabilities $P_{\bullet, 0}$ and $P_{\bullet, 1}$ are

$$
P_{\bullet, 0}=\frac{(\phi+\xi)\left(\mu\left(\eta C-\xi P_{0,0}\right)-\lambda \eta C\right)}{\phi C(\phi \mu+\xi(\mu-\lambda))}
$$

and

$$
P_{\bullet, 1}=\frac{\lambda \phi \eta C+\xi \mu(\phi+\xi) P_{0,0}}{\eta C(\mu \phi+\xi(\mu-\lambda))}
$$

where

$$
P_{0,0}=\frac{\phi \eta C(\phi+\xi)(\mu-\lambda)}{\left(\mu \phi \eta+\mu \eta \xi-\lambda \eta \xi+\mu \phi^{2}+\mu \phi \xi\right) \xi}
$$

and

$$
C=\int_{0}^{1} \mathrm{e}^{-\lambda x / \xi}(1-x)^{(\phi / \xi)-1} d x
$$

which coincide with equations (17) and (18) of Padmavathy et al. [15].

When $v=0, \eta \rightarrow \infty, \theta=1, \alpha=1$, and $\beta=1$, then, the steady-state-probabilities of the number of customers in the system have the following forms: $P_{\bullet, j}=A^{j-1} P_{0,0}$, $j=\overline{0, K-1}$, and

$$
P_{\bullet, K}=\frac{\phi}{\mu-\lambda}\left(\frac{\lambda\left(1-A^{K}\right)}{(\phi+\xi) A(1-A)}+\frac{\mu}{\lambda} A^{K-1}\right) P_{0,0}
$$

where

$$
P_{0,0}=\left(\frac{(\mu \phi+(\mu-\lambda) \xi)\left(1-A^{K}\right)}{(\mu-\lambda)(\phi+\xi) A(1-A)}+\frac{\mu \phi A^{K-1}}{\lambda(\mu-\lambda)}\right)^{-1}
$$


with

$$
A=\frac{\phi C}{\xi}
$$

such that

$$
C=\int_{0}^{1} \mathrm{e}^{-\lambda x / \zeta}(1-x)^{(\phi / \zeta)-1} d x
$$

These coincide with equations (26) and (33) of Yue et al. [31].

\section{Cost model}

We develop a model for the costs incurred in the suggested queueing model under multiple vacation policy. The following cost elements are needed:

- $C_{1}-$ cost per unit time when the server is serving customers during regular busy period,

- $C_{2}-$ cost per unit time when the server is on working vacation,

- $C_{3}-$ cost per unit time when the server is idle during regular busy period,

- $C_{4}-$ cost per unit time when a customer joins the system,

- $C_{5}-$ cost per unit time when a customer balks,

- $C_{6}-$ cost per service per unit time,

- $C_{7}-$ cost per unit time when a customer reneges,

- $C_{8}-$ cost per unit time when a customer is retained, either during busy or vacation period,

- $C_{9}-$ cost per unit time when a customer returns to the system as a feedback customer.

The total expected cost per unit time of the system is given as:

$$
\Gamma=C_{1} P_{b}+C_{2} P_{w v}+C_{3} P_{i d}+C_{4} E\left(L_{s}\right)+C_{5} R_{b}+C_{7} R_{r e n}+C_{8} R_{r e t}+(\mu+v)\left(C_{6}+\bar{\beta} C_{9}\right)
$$

\section{Numerical analysis}

\subsection{Cost optimisation}

We consider the cost optimisation problem under a given cost structure via a quadratic fit search method (QFSM). We draw attention to the optimisation of the service rates $\mu$ 
and $v$ to minimise the cost function $\Gamma$. In the previous section, a total expected cost function is developed to determine optimum regular service rates $\left(\mu^{*}, v^{*}\right)$ and the optimum expected cost $\Gamma\left(\mu^{*}, v^{*}\right)=\Gamma^{*}$. Suppose that all system parameters have fixed values, and the only controlled parameters are $\mu$ and $v$. The cost minimisation problem can be given as

$$
\min _{\mu, \nu} \Gamma(\mu, v)
$$

For computational examples, we take the cost elements as follows: $C_{1}=3, C_{2}=1$, $C_{3}=1, C_{4}=3, C_{5}=1, C_{7}=1, C_{8}=1, C_{6}=1$, and $C_{9}=1$. The expected total cost function is plotted, using QFSM, in Figs. 2-9, by varying values of $K, \xi, \phi$, and $\eta$, respectively.

- Figures 2 and 3 present the minimum values of $\mu$ and $v$ along with $\Gamma\left(\mu^{*}, v^{*}\right)$ for $K=11$ and $K=12$, respectively. The other parameters are chosen as $\lambda=11, \beta=0.8, \alpha=0.8$, $\xi=1, \phi=3, \eta=1, \theta=0.6$.

- Figures 4 and 5 display the minimum values of $\mu$ and $v$ along with $\Gamma\left(\mu^{*}, v^{*}\right)$ for $\xi=0.1$ and $\xi=2$, respectively. The other parameters are chosen as $\lambda=11, \beta=0.8$, $\alpha=0.8, \phi=3, \eta=1, \theta=0.6$, and $K=11$.

- Figures 6 and 7 depict the minimum values of $\mu$ and $v$ along with $\Gamma\left(\mu^{*}, v^{*}\right)$ for $\phi=3$ and $\phi=4$, respectively. The other parameters are chosen as $\lambda=11, \beta=0.8, \alpha=0.8, \eta=1$, $\theta=0.6$, and $K=11$.

- Figures 8 and 9 show the minimum values of $\mu$ and $v$ along with $\Gamma\left(\mu^{*}, v^{*}\right)$ and for $\eta=0.6$ and $\eta=1.4$, respectively. The other parameters are chosen as $\lambda=11, \beta=0.8$, $\alpha=0.8, \xi=1, \phi=3, \theta=0.6$, and $K=11$.

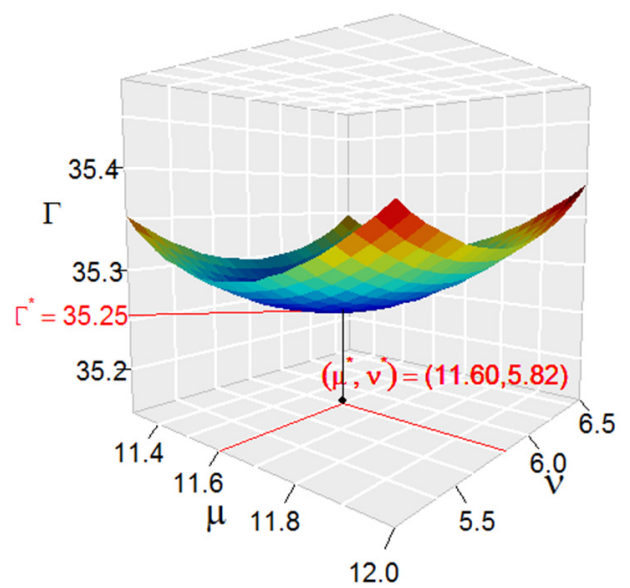

Fig. 2. The optimal values $\mu^{*}, v^{*}$ and $\Gamma^{*}$ for $K=11$ 


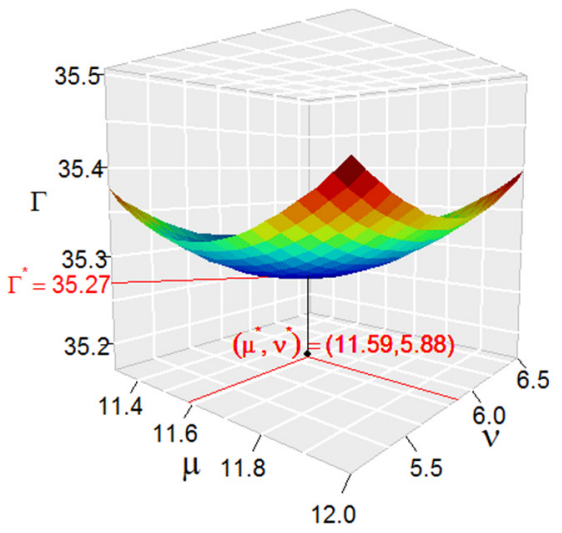

Fig. 3. The optimal values $\mu^{*}, v^{*}$ and $\Gamma^{*}$ for $K=12$

From Figs. 2-9, we see the convexity of the curves, which shows that there exist certain values of the service rates $\mu$ and $v$ that minimise the total expected cost function for the chosen set of model parameters for different values of $K, \xi, \phi$, and $\eta$. It is worth pointing out that we had to choose the values for the parameters in such a way that the stability condition $\theta \lambda<\beta \mu$ is verified.

From Figs. 2, 3, we observe that for $K=11$, the minimum expected operating cost per unit time converges to the solution $\Gamma=35.25$ at $\mu^{*}=11.60$ and $v^{*}=5.82$. For $K=12$, the minimum expected operating cost per unit time converges to the solution $\Gamma=35.27$ at $\mu^{*}=11.59$ and $v^{*}=5.88$; the minimum expected cost $\Gamma\left(\mu^{*}, v^{*}\right)$ for $K=11$ is smaller than $\Gamma\left(\mu^{*}, v^{*}\right)$ when $K=12$. This is because when $K=11$, the mean service time is bigger than that the mean service time in case of $K=12$. Further, it is quite clear that when the number of a successive number of vacation is small, customers are served faster, which generates an empty queue. This leads to a decrease in the expected cost function.

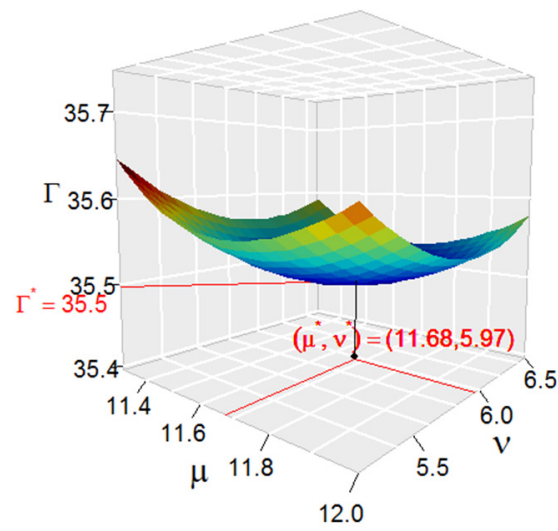

Fig. 4. The optimal values $\mu^{*}, v^{*}$ and $\Gamma^{*}$ for $\xi=0.1$ 


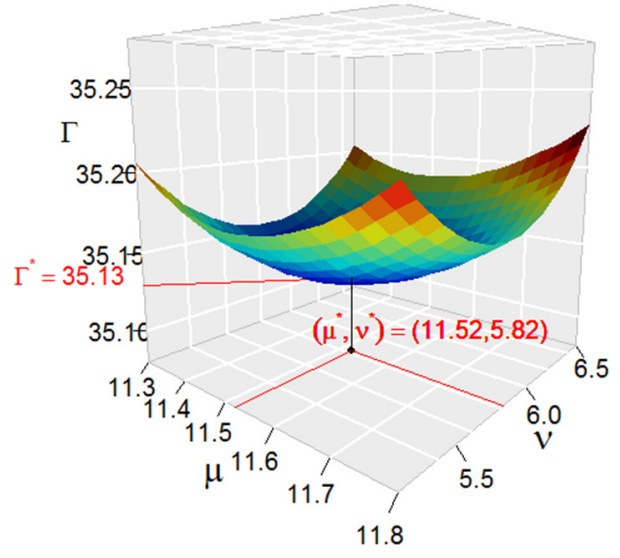

Fig. 5. The optimal values $\mu^{*}, v^{*}$ and $\Gamma^{*}$ for $\xi=2$

From Figs. 4, 5 we observe that for $\xi=0.1$, the minimum expected operating cost per unit time converges to the solution $\Gamma=35.50$ at $\mu^{*}=11.68$ and $v^{*}=5.97$. For $\xi=2$, the minimum expected operating cost per unit time converges to the solution $\Gamma=35.13$ at $\mu^{*}=11.52$ and $v^{*}=5.82$. Besides, from Figs. 2, 4, 5, we have that the minimum expected $\operatorname{cost} \Gamma\left(\mu^{*}, v^{*}\right)$ for $\xi=0.1$ is greater than $\Gamma\left(\mu^{*}, v^{*}\right)$ when $\xi=2$ and $\xi=1$. The increase in the impatience rate $\xi$ engenders a decrease in the system size, which implies a diminution in the expected cost function.

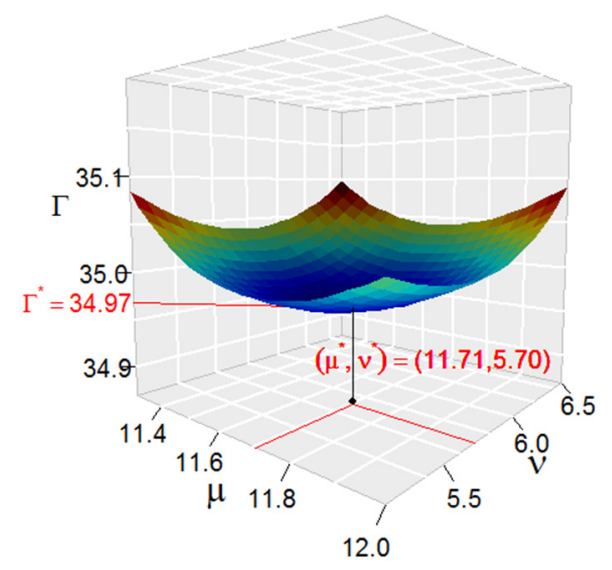

Fig. 6. The optimal values $\mu^{*}, v^{*}$ and $\Gamma^{*}$ for $\phi=4$

- From Figs. 6, 7, we observe that for $\phi=4$, the minimum expected operating cost per unit time converges to the solution $\Gamma=34.97$ at $\mu^{*}=11.71$ and $v^{*}=5.70$. For $\phi=5$, the minimum expected operating cost per unit time converges to the solution $\Gamma=34.84$ 
at $\mu^{*}=11.78$ and $v^{*}=5.66$. Further, the minimum expected cost $\Gamma\left(\mu^{*}, v^{*}\right)$ for $\phi=4$ is greater than that of and $\phi=5$ and smaller than that of $\phi=1$ (Figs. 2, 5, 6). The increase of vacation rate $\phi$ implies a decrease in the mean number of customers in the system which, in turn, decreases the expected cost function.

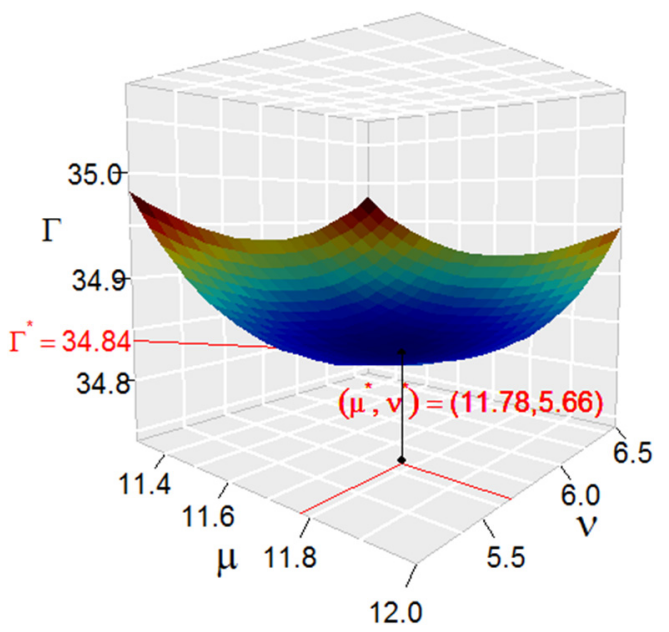

Fig. 7. The optimal values $\mu^{*}, v^{*}$ and $\Gamma^{*}$ for $\phi=5$

- From Figs. 8, 9, we observe that for $\eta=0.6$, the minimum expected operating cost per unit time converges to the solution $\Gamma=35.39$ at $\mu^{*}=11.53$ and $v^{*}=5.66$. For $\eta=1.4$, the minimum expected operating cost per unit time converges to the solution $\Gamma=35.80$ at $\mu^{*}=11.71$ and $v^{*}=5.66$.

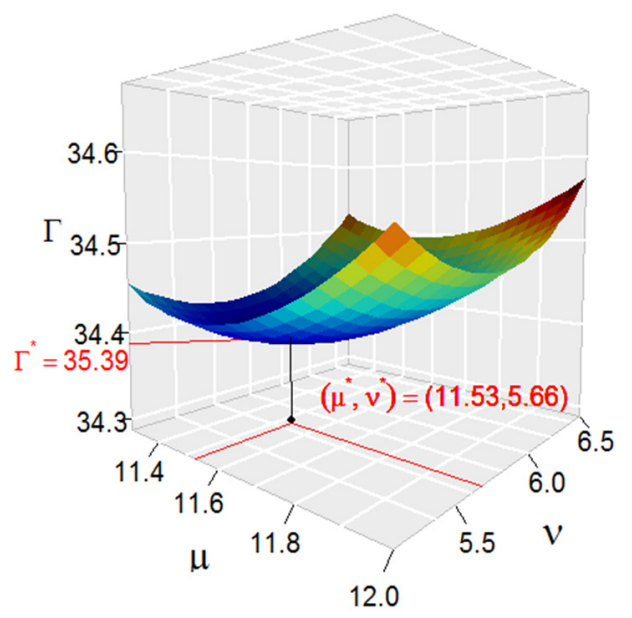

Fig. 8. The optimal values $\mu^{*}, v^{*}$ and $\Gamma^{*}$ for $\eta=0.6$ 


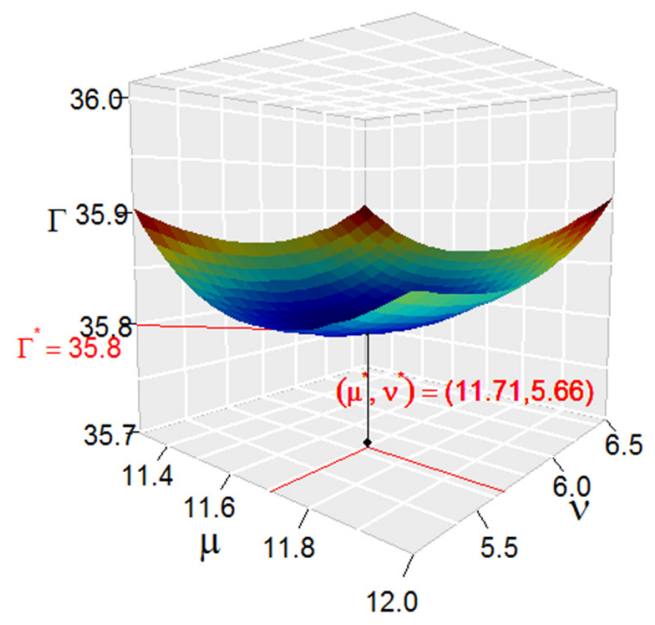

Fig. 9. The optimal values $\mu^{*}, v^{*}$ and $\Gamma^{*}$ for $\eta=1.4$

Moreover, the minimum expected cost $\Gamma\left(\mu^{*}, v^{*}\right)$ for $\eta=0.6$ is smaller than that when $\eta=1.4$. Clearly, with the increase of the waiting server $\eta$ the server switches to the working vacation quickly, which negatively affects the cost of the system which increases accordingly.

\subsection{Performance measures study}

Diverse performance measures are presented numerically. These measures are obtained by developing a program in $\mathrm{R}$ software. To this end, we put put $\lambda=11, \beta=0.8, \mu=10$, $v=6, \alpha=0.8, \xi=1, \phi=3, \eta=1, \theta=0.6$, and $K=11$. From Table 1, we have:

- The increase of the arrival rate $\lambda$ engenders a high number of customers in the systems which generates a high probability of regular busy period $P_{b}$ and small working vacation probability $P_{w v}$. The probability that the server is idle during regular busy period $P_{i d}$ decreases. This generates a large mean number of customers served as well as a mean number of lost customers.

- The increase of vacation rate $\phi$ means that the server is rapidly switching to the regular busy period at which customers are served with a higher rate. This implies an increase in $P_{b}$ and $P_{i d}$ and a decrease in $P_{w v}$ which, in turn, leads to the augmentation of the mean number of customers served $S_{r}$. Consequently, the mean number of customers in the system $E\left(L_{s}\right)$ ), as well as the mean number of loss customers $L_{r}$, diminish significantly.

- The increase of $\eta$ means that once the regular busy period ends, the server does not have much time in waiting for new arrivals, $P_{i d}$ decreases. Then, $P_{b}$ decreases while $P_{w v}$ increases, which implies an increase in $E\left(L_{s}\right)$. In this situation, more customers get 
impatient and leave the system ( $L_{r}$ increases) without getting service $\left(S_{r}\right.$ decreases). Therefore, the probability of having 0 customers in the system $P_{0,0}$ grows.

Table 1. Effect of of $\lambda, \eta, \mu, v, \phi, \xi, K$ and $\beta$ on performance measures

\begin{tabular}{|c|c|c|c|c|c|c|c|c|}
\hline Parameter & & $P_{0,0}$ & $P_{w v}$ & $P_{b}$ & $P_{i d}$ & $E\left(L_{S}\right)$ & $L_{r}$ & $S_{r}$ \\
\hline \multirow{3}{*}{$\lambda$} & 10 & 0.027 & 0.120 & 0.755 & 0.125 & 2.7261 & 3.362 & 6.171 \\
\hline & 11 & 0.019 & 0.077 & 0.831 & 0.091 & 3.865 & 3.911 & 6.738 \\
\hline & 12 & 0.010 & 0.041 & 0.905 & 0.054 & 6.730 & 4.499 & 7.291 \\
\hline \multirow{3}{*}{$\phi$} & 0.1 & 0.122 & 0.548 & 0.415 & 0.036 & 5.406 & 5.81 & 3.910 \\
\hline & 0.2 & 0.088 & 0.389 & 0.561 & 0.051 & 5.126 & 5.14 & 4.909 \\
\hline & 0.3 & 0.070 & 0.307 & 0.634 & 0.059 & 4.889 & 4.79 & 5.407 \\
\hline \multirow{3}{*}{$\eta$} & 0.1 & 0.003 & 0.012 & 0.845 & 0.143 & 2.910 & 3.759 & 6.776 \\
\hline & 0.2 & 0.005 & 0.023 & 0.843 & 0.134 & 3.066 & 3.784 & 6.770 \\
\hline & 0.3 & 0.008 & 0.032 & 0.841 & 0.127 & 3.204 & 3.806 & 6.764 \\
\hline \multirow{3}{*}{$\xi$} & 1 & 0.019 & 0.077 & 0.831 & 0.091 & 3.865 & 3.911 & 6.738 \\
\hline & 2 & 0.020 & 0.086 & 0.822 & 0.092 & 3.754 & 3.982 & 6.671 \\
\hline & 3 & 0.021 & 0.095 & 0.814 & 0.092 & 3.677 & 4.052 & 6.608 \\
\hline \multirow{3}{*}{$\mu$} & 11 & 0.028 & 0.125 & 0.752 & 0.123 & 2.932 & 3.698 & 6.747 \\
\hline & 12 & 0.034 & 0.176 & 0.687 & 0.138 & 2.581 & 3.536 & 6.748 \\
\hline & 13 & 0.037 & 0.227 & 0.636 & 0.136 & 2.454 & 3.425 & 6.773 \\
\hline \multirow{3}{*}{$n$} & 5 & 0.019 & 0.087 & 0.829 & 0.084 & 4.377 & 3.922 & 6.707 \\
\hline & 6 & 0.019 & 0.077 & 0.831 & 0.091 & 3.865 & 3.911 & 6.738 \\
\hline & 7 & 0.017 & 0.070 & 0.836 & 0.095 & 3.435 & 3.910 & 6.782 \\
\hline \multirow{3}{*}{$K$} & 7 & 0.019 & 0.076 & 0.832 & 0.092 & 3.847 & 3.908 & 6.738 \\
\hline & 9 & 0.019 & 0.077 & 0.831 & 0.092 & 3.860 & 3.911 & 6.738 \\
\hline & 11 & 0.019 & 0.077 & 0.831 & 0.091 & 3.865 & 3.911 & 6.738 \\
\hline \multirow{3}{*}{$\beta$} & 0.75 & 0.012 & 0.051 & 0.886 & 0.063 & 5.649 & 4.070 & 6.702 \\
\hline & 0.85 & 0.024 & 0.103 & 0.781 & 0.116 & 3.029 & 3.768 & 6.764 \\
\hline & 0.95 & 0.034 & 0.151 & 0.695 & 0.155 & 2.239 & 3.521 & 6.791 \\
\hline
\end{tabular}

- With the increase in reneging rate $\xi$, the mean number of customers in the systems during working vacation period decreases $E\left(L_{s}\right)$, which generates a high probability of having 0 customers in the system $P_{0,0}$. This generates an increase in the mean number of loss customers $L_{r}$. Further, the augmentation of $\xi$ implies an increase in $P_{w v}$ and $P_{i d}$, and a decrease in $P_{b}$, which, in turn, yields a decreasing in the mean number of served customers $S_{r}$. Also, the increases in the impatience rate imply a decrease in the mean number of customers in the system. Therefore, the arriving customers enter significantly to the system ( $R_{b}$ decreases). Nevertheless, the increase of $\xi$ means that the system is losing many customers. In this situation, using a certain mechanism, the latter may be convinced to stay in the queue ( $R_{r e t}$ increases).

- With the increase of the service rate during regular busy period $\mu$, the mean number of customers in the system increases, which implies a decrease in the probability of 
regular busy period and an increase in the probability that the sever gets idle just after a regular busy period is ended as well as the working vacation probability $P_{w v}$.

- As the service rate during the vacation period $v$ increases, the mean number of customers in the system decreases. Consequently, the mean number of lost customers decreases, as intuitively expected. Further, the probability that the server switches to the regular busy period at which the customers are served with faster rate increases. This leads to an augmentation in the mean number of customers served.

- The increases in $K$ implies an increase in $P_{w}$. This leads to an increase in $E\left(L_{s}\right)$ which, in turn, increases $L_{r}$. The other performance measures $P_{w v}, P_{i d}$ decrease. The mean number of customers served $S_{r}$ and $P_{0,0}$ are stable with $K$, which can be due to the choice of the system parameters.

- With the increasing of $\beta$, we observe an increasing trend in $P_{i d}, P_{0,0}, P_{w v}, E\left(L_{s}\right)$, and $S_{r}$. Further, a decreasing trend is seen in $P_{b}$ and $L_{r}$ with $\beta$.

- As intuitively expected, the increase of $\alpha$ generates a high average rate of reneging $R_{r e n}$ which, in turn, implies a decrease in the mean number of customers in the system $E\left(L_{s}\right)$. This yields a decrease in the average rate of balking $P_{b}$.

- The increases of $\theta$ imply a decreasing of $R_{b}$ and an increase $S_{r}$. Obviously, the larger the probability of joining the system, the smaller the average rate of balking. Besides, when the probability of joining the system augments, the mean number of customers in the system becomes large. This implies an increase in the mean number of customers served.

- The increase of the $\alpha$ generates a significant mean number of loss customers which, in turn, implies a decrease in the mean number of customers served. This yields a decrease in the average rate of balking.

- With the increase of the probability of joining the system $\theta$, the mean number of customers served increases, this engenders a high probability of a busy period. This, in turn, generates a large mean number of customers served $S_{r}$.

\section{Conclusion}

In this investigation, we analyse a single server Markovian queueing model with Bernoulli feedback, $K$-variant working vacation policy, balking, reneging, and retention of reneged customers wherein at the end of the regular busy period the server has to wait before it goes on a working vacation. The probability generating functions for the numbers of customers in the system are obtained. Then, explicit expressions for useful system performance measures are derived. Moreover, the stochastic decomposition theorems are established. After that, some special cases of the considered queueing system are presented. A cost model is developed. Further, a cost optimisation is carried 
out, using a quadratic fit search method (QFSM). Finally, the analytical results are validated with some numerical examples, which may be useful in diverse real-life situations to design the outputs. The motivation for the suggested model comes from large applications in many real-time systems, including computer and communication networks.

Hopefully, this work will be of great help to system managers. This study can be extended in many directions by incorporating the concepts of batch arrival and breakdowns with repairs.

\section{References}

[1] AMMAR S.I., Transient solution of an vacation queue with a waiting server and impatient customers, J. Egypt. Math. Soc., 2017, 25, 337-342.

[2] AZHAGAPPAN A., Transient behavior of a Markovian queue with working vacation variant reneging and a waiting server, TOP, 2019, 27, 351.

[3] Bouchentouf A.A., Guendouzi A., Cost optimization analysis for an vacation queueing system with waiting servers and impatient customers, SeMA, 2019, 76, 309-341.

[4] Bouchentouf A.A., Guendouzi A., The Bernoulli feedback queue with variant multiple working vacations and impatient customers: Performance and economic analysis, Arab. J. Math., 2019, DOI: 10.1007/s40065- 019-0260-x, 1-19.

[5] Bouchentouf A.A., Guendouzi A., Kandouci A., Performance and economic study of heterogeneous $M / M / 2 / N$ feedback queue with working vacation and impatient customers, ProbStat Forum, 2019, 12 (1), 15-35.

[6] Bouchentouf A.A., Cherfaoui M., Bounlem M., Performance and economic analysis of a single server feedback queueing model with vacation and impatient customers, OPSEARCH, 2019, 56 (1), $300-320$.

[7] Bouchentouf A.A., Messabini A., Heterogeneous two-server queueing system with reverse balking and reneging, OPSEARCH, 2018, 55 (2), 251-267.

[8] BouCHENTOUF A.A., YAHIAOUI L., On feedback queueing system with reneging and retention of reneged customers, multiple working vacations and Bernoulli schedule vacation interruption, Arab. J. Math., 2017, 6 (1), 1-11.

[9] Choudhury G., Paul M., A two phase queueing system with Bernoulli feedback, J. Inf. Manage. Sci., 2005, 16 (1), 35-52.

[10] DeEPA B., KALIDASS K., The Markovian vacation queues with a waiting server and geometric abandonments, Int. J. Pure Appl. Math., 2018, 118, 1903-1910.

[11] LAXмI V.P., JYOTHSNA K., Analysis of finite buffer renewal input queue with balking and multiple working vacations, OPSEARCH, 2013, 50 (4), 548-565.

[12] LAXMI V.P., RAJESH P., Analysis of variant working vacations queue with customer impatience. Int. J. Manage. Sci. Eng. Manage., 2016, 12, 186-195.

[13] LAXMI V.P., RAJeSh P., Performance measures of variant working vacation on batch arrival queue with reneging, Int. J. Math. Arch., 2017, 8, 85-96.

[14] Li J., TiAn N., The M/M/1 queue with working vacations and vacation interruptions, J. Syst. Sci. Syst. Eng., 2007, 16 (1), 121-127.

[15] Padmavathy R., Kalidass K., RAmAnAth K., Vacation queues with impatient customers and a waiting server, Int. J. Latest Trends Soft. Eng., 2011, 1, 10-19.

[16] KALIDASS K., KASTURI R., A two phase service $M / G / 1$ queue with a finite number of immediate Bernoulli feedbacks, OPSEARCH, 2014, 51 (2), 201-218. 
[17] Kalidass K., Ramanath K., Time dependent analysis of queue with server vacations and a waiting server, QTNA 2011, Proc. 6th International Conference on Queueing Theory and Network Applications, Korea University, 2011, 77-83, https://doi.org/10.1145/2021216.2021227

[18] KeILSON J., SERVi L.D., A distribution form of Littles law, Oper. Res. Lett., 1988, 7 (5), 223-227.

[19] Krishna Kumar B., ViJayakumar A., Arivudainambi D., The M/G/1 retrial queue with Bernoulli schedules and general retrial times, Comp. Math. Appl., 2002, 43, 15-30.

[20] Selvaraju N., Goswami C., Impatient customers in an M/M/1 queue with single and multiple working vacations, Comp. Ind. Eng., 2013, 65, 207-215.

[21] SERVi L.D., FinN S.G., M/M/1 queues with working vacations (M/M/1/WV), Perf. Eval., 2002, 50, 41-52.

[22] Shakir M., MANOHARAn P., Analysis of the M/M/1 queue with single working vacation and vacation interruption (IJMTT), Int. J. Math. Trends Techn., 2017, 47 (1), 32-40.

[23] SHAKIR M., MANOHARAN P., Analysis of a M/M/c queue with single and multiple synchronous working vacations, Appl. Appl. Math., 2017, 12 (2), 671-694.

[24] ShakiR M., MANOHARAN P., Impatient customers in an M/M/c queue with single and Multiple Synchronous Working Vacations, Pakistan J. Stat. Oper. Res., 2018, 14 (3), 571-594.

[25] Sudhesh R., AzHAGappan A., Transient analysis of an M/M/1 queue with variant impatient behavior and working vacations, OPSEARCH, 2018, 55 (3-4), 787-806.

[26] SUDHESH R., RAJ L.F., Computational analysis of stationary and transient distribution of single server queue with working vacation, Global Trends Comp. Comm. Syst. Comm. Comp. Inf. Sci., 2012, 269, 480-489.

[27] Tian N., ZhaO X., WAng K., The M/M/1 queue with single working vacation, Int. J. Inf. Manage. Sci., 2008, 19, 621-634.

[28] TaKacs L., A Single Server Queue with Feedback, Bell Syst. Tech. J., 1963, 42, 505-519.

[29] YahiaOui L., Bouchentouf A.A., KADi M., Optimum cost analysis for an feedback queue under synchronous working vacations and impatient customers, Croatian Operational Research Review, 2019, 10, 211-226.

[30] YeChiali U., On the queue with a waiting server and vacations, Sankhya: Indian J. Stat., 2004, 66 (1), 159-174.

[31] Yue D., Yue W., SAFFer Z., Chen X., Analysis of an queueing system with impatient customers and a variant of multiple vacation policy, J. Ind. Manage. Opt., 2014, 10, 89-112.

[32] Yue D., Yue W., Xu G., Analysis of a queueing system with impatient customers and working vacations, Proc. 6th International Conference on Queueing Theory and Network Applications, Korea University, 2011, 208-211.

[33] Varalakshmi M., Chandrasekaran V.M., Saravanarajan M.C., A single server queue with immediate feedback, working vacation and server breakdown, Int. J. Eng. Techn., 2018, 7 (4.10), 476479.

[34] Varalakshmi M., Saravanarajan M.C., ChandraseKaran V.M., A study on $M / G / 1$ retrial G-queue with two phase of service, immediate feedback and working vacation, IOP Conference Series, Materials Science and Engineering, 2017, 263, 042156.

[35] Wang T.Y., Ke J.C., Chang F.M., On the discrete-time queue with randomized vacations and at most vacations, Appl. Math. Model., 2011, 35, 2297-2308.

[36] Zhang Z.G., TiAn N., Discrete time queue with multiple adaptive vacations, Queueing Syst., 2001, $38,419-429$. 